\title{
Effects of vibrational energy relaxation and reverse reaction on electron transfer kinetics and fluorescence line shapes in solution
}

\author{
R. Aldrin Denny a) and Biman Bagchib) \\ Solid State and Structural Chemistry Unit, Indian Institute of Science, Bangalore 560 012, India \\ Paul F. Barbara \\ Department of Chemistry and Biochemistry, The University of Texas at Austin, Austin, Texas 78712
}

\begin{abstract}
The existing theoretical formulations of electron transfer reactions (ETR) neglect the effects of vibrational energy relaxation (VER) and do not include higher vibrational states in both the reactant and the product surfaces. Both of these aspects can be important for photo-induced electron transfer reactions, particularly for those which are in the Marcus inverted regime. In this article, a theoretical formulation is presented which describes the two aspects. The formalism requires an extension of the hybrid model introduced earlier by Barbara et al. [Science 256, 975 (1992)]. We model a general electron transfer as a two-surface reaction where overlap between the vibrational levels of the two surfaces create multiple, broad reaction windows. The strength and the accessibility of each window is determined by many factors. We find that when VER and reverse transfer are present, the time dependence of the survival probability of the reactant differs significantly (from the case when they are assumed to be absent) for a large range of values of the solvent reorganization energy $\left(\lambda_{X}\right)$, quantum mode reorganization energy $\left(\lambda_{q}\right)$, electronic coupling constant $\left(V_{\mathrm{el}}\right)$ and vibrational energy relaxation rate $\left(k_{\mathrm{VER}}\right)$. Several interesting results, such as a transient rise in the population of the zeroth vibrational level of the reactant surface, a Kramers (or Grote-Hynes) type recrossing due to back reaction and a pronounced role of the initial Gaussian component of the solvation time correlation function in the dynamics of electron transfer reaction, are observed. Significant dependence of the electron transfer rate on the ultrafast Gaussian component of solvation dynamics is predicted for a range of values of $V_{\mathrm{el}}$, although dependence on average solvation time can be weak. Another result is that, although VER alters relaxation dynamics in both the product and the reactant surfaces noticeably, the average rate of electron transfer is found to be weakly dependent on $k_{\mathrm{VER}}$ for a range of values of $V_{\mathrm{el}}$; this independence breaks down only at very small values of $V_{\mathrm{el}}$. In addition, the hybrid model is employed to study the time resolved fluorescence line shape for the electron transfer reactions. It is found that VER can have a significant influence on the fluorescence spectrum. The possibility of vibrational state resolved spectra is investigated.
\end{abstract}

\section{INTRODUCTION}

Electron transfer reactions between donor (D)-acceptor (A) pairs in solution and in organized media exhibit diverse behavior much of which can be rationalized within the wellknown and well-tested Marcus theory. ${ }^{1-5}$ Zusman, ${ }^{6}$ Fonseca, ${ }^{7}$ and $\mathrm{Hynes}^{8}$ extended this theory to treat the dynamics of electron transfer reaction and to investigate the role of solvation dynamics in adiabatic electron transfer. The Zusman-Hynes formulation, which predicted too strong a solvent relaxation dependence in some cases, was further extended by Sumi and Marcus, ${ }^{9}$ who included the role of a vibrational coordinate to explain the observed lack of solvent relaxation dependence of the ETR rate in some systems. Many of these aspects have been summarized and reviewed recently. ${ }^{10-19}$

\footnotetext{
a) Present address: Department of Chemistry and Chemical Biology, Harvard University, Cambridge, Massachusetts 02138.

${ }^{b)}$ Author to whom correspondence should be addressed. Electronic mail: bbagchi@sscu.iisc.ernet.in
}

Photo-induced electron transfer reactions, however, sometimes show behavior which is at variance with Marcus theory. ${ }^{20-23}$ This is particularly true for those photo-induced reactions which occur in the Marcus inverted regime- the observed rate is often much larger than the prediction of the Marcus theory. ${ }^{19,24-27}$ Jortner and Bixon ${ }^{28-30}$ proposed that the explanation of this remarkable behavior can be found in the participation of high frequency quantum modes which can open up additional reaction channels in the barrierless and even in the normal region, so that the slow activation process earlier deemed necessary for the inverted reactions is not required. The ideas of Sumi and Marcus and of Jortner and Bixon were subsequently combined in a "hybrid model" by Barbara and co-workers. ${ }^{26,27,31}$ The hybrid model is a minimal model which envisages an electron transfer to occur on a three-dimensional surface spanned by the solvent polarization coordinate $(X)$, a low frequency classical vibrational coordinate $(Q)$ and a high frequency vibration $(q)$, to be treated quantum mechanically. Since the choice of the vibrational coordinates is not very clear, they are obtained by 
fitting to the absorption spectrum. In the applications of the hybrid model, it has always been assumed that the relaxation of both the vibrational modes is much faster than the electron transfer rate, so that the effects of these two modes are manifested in the location and width of the multiple reaction windows. This model has been shown to exhibit rich and diverse, behavior and is a good candidate which could potentially explain a number of yet unexplained results. ${ }^{19,26,27,31}$

In photo-induced electron transfer reactions (ETRs), the initial excited states are often the vibrationally hot states. $^{31-38}$ The efficient reaction windows, however, are usually located near the minimum of the reactant surface. Thus, vibrational energy relaxation and redistribution must occur before electron transfer can take place. As already pointed out, vibrational energy relaxation of these hot states has been neglected in the existing discussions. It is amusing to note that there seems to exist two diagonally opposite rationalizations for the neglect of vibrational energy relaxtion (VER). First, of course, is the assumption that VER of these hot states is much faster than electron transfer. Thus, ETR always occurs from the vibrational ground state of the reactant. ${ }^{9}$ The problem with this assumption is that for fast ETRs, this separation of time scales may not exist. The other view is that VER is quite slow and ETR occurs from the initially populated, vibrationally unrelaxed distribution function. When efficient sinks are present all around the initially excited state, VER (and even the solvation energy relaxation) is not essential for ETR to occur with high speed. This has been the view of Jortner and Bixon. ${ }^{28-30}$ The difficulty with this explanation is that one requires participation of several high frequency vibrational modes to give rise to such high density of reaction windows. In the absence of reliable numbers for the rates of VER in systems undergoing ETR, it is hard to justify any of the two scenarios. The real situation may lie in between the above two extremes. A good example of this may be the back electron transfer in the excited betaine-30 which has been studied extensively by Barbara and co-workers. ${ }^{26,27,31}$ In this case, the system is optically prepared in a vibrationally hot state.

In an interesting piece of work, Sparpaglione and Mukamel $^{39}$ rederived Zusman's reaction-diffusion result using a master equation approach, where the solvent is treated completely quantum mechanically. The advantage of this method is shown by Makri et al. ${ }^{40,41}$ by performing a path integral simulation considering a nonexponential short-time behavior of the electronic population. Coalson et al., ${ }^{42-44}$ Nitzan, ${ }^{45}$ and others ${ }^{46}$ extended this fully quantum mechanical theory to handle anharmonic environments, the nonequilibrium nuclear initial preparation and back electron flow. Numerical calculations of these models reveal that most of them are numerically unstable, and thus, extension of them to incorporate VER when reactions occur in higher vibrational states is highly nontrivial. In addition, when the electronic coupling matrix element is small and the nonadiabatic formulation of ETR is appropriate, the back electron transfer is significant. A few works have considered this aspect. ${ }^{44,47}$ However, while one can easily see this effect on the average rate in a rate law description, the effects of back electron transfer on the detailed time dependence of survival probability have not been investigated. That is, for nonexponential kinetics, it is not clear what part of the survival probability is most effected.

Both these effects (VER and back reaction) find additional significance in view of the discovery made in the last decade that the solvation time correlation function in many common polar solvents (like water and acetonitrile) is biphasic, with about $60 \%-70 \%$ of solvation occurring in less than 100 femtoseconds (fs). ${ }^{48-50}$ This ultrafast solvation is usually followed by a slow decay with a time constant in the few picosecond (ps) range. This biphasic solvation can significantly affect the role of VER and reverse reaction. For example, while the average electron transfer time, $\left\langle\tau_{\text {et }}\right\rangle$, can be weakly dependent on the average solvation time $\left\langle\tau_{s}\right\rangle$, a stronger dependence of $\left\langle\tau_{\text {et }}\right\rangle$ on the initial ultrafast component cannot be ruled out. Note that the studies carried out in the late eighties and early nineties ignored the presence of the ultrafast component and, therefore, might have reached erroneous conclusions regarding the relation between electron transfer rate and solvation dynamics.

Fluorescence from a photoexcited molecule participating (or undergoing) an electron transfer reaction has been a time honored technique to obtain the rate of decay of the emitting state. $^{51-54}$ The disappearance of the integrated fluorescence intensity yields the rate of the electron transfer reaction. The study of the line shape, on the other hand, can provide more detailed information, ${ }^{53}$ although such studies are less frequent, because of the difficulty of probing the emission spectrum at many wavelengths. Theoretical and computational studies can be useful in this case. Barbara and co-workers ${ }^{26,27,31}$ have already studied the time dependence of fluorescence spectrum. However, no study of the effects of VER on emission spectrum has ever been reported.

Theoretical study of electron transfer rates in such a multidimensional potential energy surface poses an interesting but formidable challenge, especially so in the presence of multiple delocalized sinks of differing reactive strengths, and a multitime scale solvent relaxation behavior. The presence of VER vastly increases the computational difficulty of the problem. The theoretical formulation presented here allows the treatment of such a complex problem in a simple fashion. We calculate not only the electron transfer dynamics (incorporating higher vibrational states in the products as well as in the reactant) but also the fluorescence line shape. This study includes both the VER and the reverse reaction, and in addition, the biphasic solvation dynamics. The model employed is a generalized hybrid model where the equation of motion now involves two coupled surfaces, each with multiple sinks; the equation of motion is a non-Markovian Smoluchowski equation, with a time dependent diffusion coefficient. The latter is related to the solvation time correlation function by a relation which is exact for harmonic surfaces.

The present study led to the following new results: The most significant result is that for a range of the electronic coupling matrix elements $V_{\mathrm{el}}$, the electron transfer rate is nearly independent of VER. This is despite the fact that the time dependence of the vibrational population distribution in the reactant and product states is significantly affected by 
VER itself. This independence breaks down only at very low values of the VER rate. Second, we find that the back electron transfer slows down the decay of the reactant survival probability time correlation function, $P_{S}(t)$, at long times, while leaving the short time part unaffected. Interestingly, this effect is nearly absent when the weight of the initial Gaussian component is negligible. Third, VER promotes electron transfer rate via the channels which are effectively in the normal regime, as the former prevents back electron transfer by removing population from the reaction zone. Fourth, the details of the initial solvation time correlation function does affect details of the time dependent survival probability, $P_{S}(t)$. For example, a Gaussian decay gives rise to a $P_{S}(t)$ which is significantly different from an exponential decay with the same time constant. In addition, our theory provides vibrational state resolved transient population distributions, both in the reactant and the product states. The fluorescence line shape is shown to depend not only on the parameters that characterize the two reaction surfaces, but also on the vibrational energy relaxation rate $\left(k_{\mathrm{VER}}\right)$, the electronic coupling element $\left(V_{\mathrm{el}}\right)$, and of course on solvation dynamics. In fact, the effects of these terms are coupled because the relaxation rates compete with electron transfer rates from the reaction sinks.

The organization of the rest of the article is as follows: In the next section we present the theoretical formulation. In Sec. III we present the numerical results along with a discussion. Section IV concludes with a brief summary.

\section{THEORETICAL FORMULATION}

We consider the charge recombination (CR) between the contact ion pair $\mathrm{A}^{+} \mathrm{B}^{-}$to form $\mathrm{AB}$. Theoretically, this is modelled as a two-surface problem (see Fig. 1). In many experiments, for example, in the case of betaines, the ground state $A B$ is optically excited, which leads to the rapid formation of $\mathrm{A}^{+} \mathrm{B}^{-}$in the ion pair state. This is denoted as the reactant, or the " $R$," state. The product state is the neutral ground state, denoted as the " $P$ " state (see Fig. 1). Subsequent to the excitation the system relaxes towards the minimum of the potential energy surface. As the charge transfer reaction is assumed to proceed on a multidimensional surface, the theoretical description usually assumes that the frequencies are all harmonic. The system is modeled by a low frequency harmonic solvent mode, a similar low frequency, harmonic, classical vibrational mode, and a high frequency harmonic quantum-mechanical vibrational mode. The potentials for the reactant and the product states are twodimensional potential energy surfaces (PES) as shown in Fig. 1. These harmonic surfaces are described by the following equations:

$$
\begin{aligned}
V_{R, m}(X, Q)= & \frac{1}{2} 2 \lambda_{X} X^{2}+\frac{1}{2} 2 \lambda_{Q} Q^{2}+m h \nu_{q}, \\
V_{P, n}(X, Q)= & \frac{1}{2} 2 \lambda_{X}(X-1)^{2}+\frac{1}{2} 2 \lambda_{Q}(Q-1)^{2}+n h \nu_{q} \\
& +\lambda_{q}+\Delta G,
\end{aligned}
$$

$V_{R, m}$ and $V_{P, n}$ denote the reactant and product states arising, respectively, from the $m$ th and $n$th vibrational level of the high-frequency quantum mode. $\Delta G$ is the free energy gap of

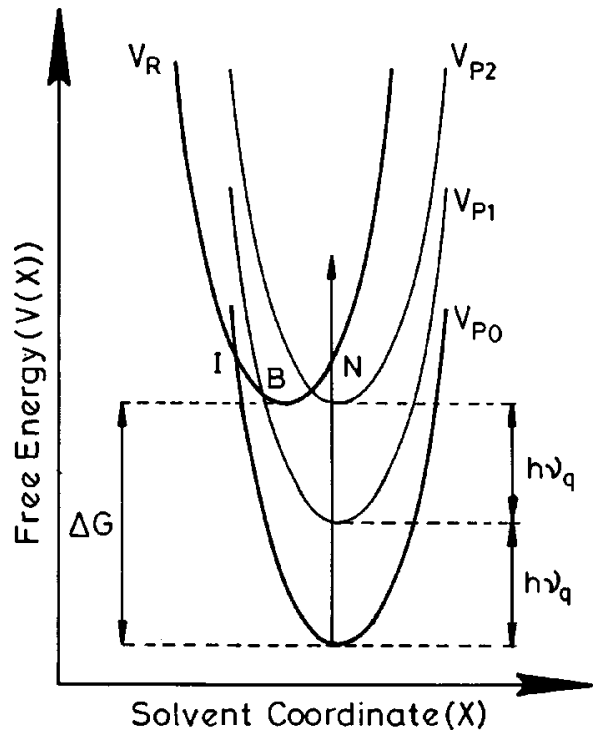

FIG. 1. A schematic representation of the two surface, multilevel, hybrid model for the electron-transfer reaction. $V_{R, m}$ and $V_{P, m}$ where $m$ and $n$ equals $0,1,2, \ldots$ are the effective potential energy surfaces for the ground reactant (or locally excited) and the product (or charge transfer) vibronic states, respectively. $\Delta G$ is the difference in the potential heights between the ground reactant $\left(V_{R, 0}\right)$ and the ground product $\left(V_{P, 0}\right)$ states. $h \nu_{q}$ is the quantum gap of the high-frequency vibrational mode. The figure is drawn in such a way that first, second and third vibrational states of the product crosses the ground reactant vibrational state at inverted (I), barrierless (B) and normal $(\mathrm{N})$ region, respectively.

the reaction. $X$ represents the solvent coordinate and is the electrostatic potential difference $e \Delta V$ between the donor and the acceptor sites, produced by the surrounding polar solvent. $Q$ and $q$ are the low frequency and the high frequency vibrational coordinates, respectively. $\lambda_{X}, \lambda_{Q}$, and $\lambda_{q}$ are the corresponding energies of reorganization of these modes. $\nu_{q}$ is the frequency of the quantum mode. A rough measure of the extent of influence of the internal vibrational modes on the dynamics is determined by the relative vibrational and solvent reorganization energies. ${ }^{9}$ When $\lambda_{X} / \lambda_{Q} \gg 1$, the sink reaction window is narrow in $X$, and the dynamics becomes solvent controlled. When $\lambda_{X} / \lambda_{Q} \ll 1$, the reaction window is broad in $X$, and the dynamics exhibits a weak $X$-dependence.

The quantum treatment of the high frequency modes can be viewed as a change in the effective free energy gap $-\Delta G_{n m} \quad\left[=(n-m) h \nu_{q}+\lambda_{q}+\Delta G\right], \quad$ between twodimensional reactant and product surfaces. A three-mode problem is now reduced to a two-mode multisurface one (Fig. 1). This approach can be easily generalized to a $m$-mode case when more than one high frequency mode is involved.

The time-evolution of the probability distribution $P_{R, i}(X, t)$ of the system on the reactant PES is assumed to be given by the following reaction-diffusion equation:

$$
\begin{aligned}
\frac{\partial P_{R, i}(X, t)}{\partial t}= & \mathcal{L}_{X} P_{R, i}(X, t)-(1+n) S(X) P_{R, i}(X, t) \\
& +S(X) P_{P}(X, t)+k_{\mathrm{VER}} P_{R, i+1}(X, t) \\
& -k_{\mathrm{VER}} P_{R, i}(X, t),
\end{aligned}
$$


where $i=0,1,2, \ldots, m$ denote the vibrational levels of the reactant surface and $n$ is the number of vibrational levels considered in the product PES. The first term describes the relaxation in the $V_{R, i}(X, Q)$ potential. The second term accounts for the actual transfer of the electron to the different product states along the sink windows. The third term describes the reverse electron transfer from the formed product state to the reactant state. The last two terms incorporate the change in the population in the vibronic levels due to the VER. Equations for $i=0$ and $i=m$ surfaces will be different from Eq. (3). In these cases terms describing the decrease and increase in population, respectively, due to the VER will be absent. A similar expression can be constructed for the $n$th surface as well. Equation (3) is a generalization of the earlier equation of motion used for hybrid model and it includes both VER and back electron transfer.

The operator $\mathcal{L}_{X}$ is the Smoluchowski operator and is given by

$$
\mathcal{L}_{X}=D_{X}(t)\left(\frac{\partial^{2}}{\partial X^{2}}+\frac{1}{k_{B} T} \frac{\partial}{\partial X}\left[\frac{\partial V(X)}{\partial X}\right]\right),
$$

where $D_{X}(t)$ is the time dependent diffusion coefficient of motion along the reaction coordinate. $D_{X}(t)$ is given by the relation $^{8}$

$$
D_{X}(t)=-k_{B} T \frac{d \ln S(t)}{\mathrm{d} t},
$$

where $S(t)$ is the solvation time correlation function of the reaction coordinate. The diffusion coefficient is time dependent when the relaxation is characterized by a multiexponential time decay (non-Markovian) and is time-independent only for a single exponential decay (Markovian). The average relaxation time $\left\langle\tau_{s}\right\rangle$ is given by

$$
\left\langle\tau_{s}\right\rangle=\int_{0}^{\infty} d t S(t) .
$$

As in earlier theoretical studies, ${ }^{9,26,27,55,56}$ the relaxation along the $Q$-mode has been assumed to be infinitely fast. In doing so it is assumed that the relaxation along the $Q$-mode effects the thermodynamics of electron transfer, thus indirectly influencing the rate. In solution, it is convenient to assume that the operator in Eq. (4) is a stochastic operator that describes the relaxation of the initial nonequilibrium population to equilibrium, with well-defined rates supplied externally. For relaxation along solvent coordinate $X$, this rate is related to the solvation rate. The solvent time correlation function $S(t)$ is defined as

$$
S(t)=\frac{\langle X(0) X(t)\rangle}{\left\langle X^{2}(0)\right\rangle},
$$

where $\langle\cdots\rangle$ denotes the average over the solvent degrees of freedom in equilibrium with the reactant state. It is the quantity $S(t)$ which reflects the dynamics of the solvent polarization fluctuations and is usually equated with the solvation time correlation function. ${ }^{8}$ In this work, we have assumed the following two forms for $S(t):^{38,47,57}$

$$
S(t)=A \exp \left(-t^{2} / \tau_{G}^{2}\right)+(1-A) \exp \left(-t / \tau_{E}\right),
$$

$$
S(t)=B \exp \left(-t / \tau_{E_{1}}\right)+(1-B) \exp \left(-t / \tau_{E}\right),
$$

where $A$ and $B$ are the prefactors in the solvation time correlation function, $\tau_{G}$ and $\tau_{E_{1}}$ are the ultrafast solvation time constants for the Gaussian and the exponential form. Note that $\tau_{G}$ and $\tau_{E_{1}}$ are much smaller than the slow component time constant $\tau_{E}$.

As the solvation time correlation function in many common dipolar liquids, especially in water and acetonitrile, is biphasic with two widely differing time constants, $D(t)$ will have a nontrivial time dependence, which may play a crucial role in the dynamics of electron transfer reactions.

The fluorescence line shape from the reactant is assumed to be given by the following well-known expression: ${ }^{58-60}$

$$
\begin{aligned}
I_{f l}(\omega, t)= & \int d X \int d Q \\
& \times \sum_{i j} P_{R}^{\mathrm{eq}}(Q) P_{R}(X, i, t)\left|V_{i j}\right|^{2} \delta(\omega-\omega(t)),
\end{aligned}
$$

where

$$
\omega(t)=\omega_{i j}(t)+\omega_{Q}(t)+\omega_{X}(t),
$$

$P_{R}^{\mathrm{eq}}(Q)$ is the equilibrium population along the classical low frequency $Q$ mode, $i$ and $j$ refer to states of light emitting and absorbing species, respectively. Note that the nontrivial problem in evaluating the line shape is the calculation of the nonequilibrium probability distribution function, $P_{R}(X, i, t)$. The calculation of $P_{R}$ will be discussed later in this section. Equation (16) essentially assumes that the vibrational levels are stable, and no fluctuations are present. However, when the energies of the vibrational levels are fluctuating with time, the expression for the nonequilibrium fluorescence line shape can be obtained by using the following expression:

$$
\begin{aligned}
& I_{n e}(\omega, t) \\
& \quad=\int d X \sum_{i j}\left|V_{i j}^{2}\right| P_{i}(X, i, t) \frac{1 / \tau_{c}}{\left(\omega-\omega_{i j}^{0}+\Delta \omega_{X}(t)\right)^{2}+\left(1 / \tau_{c}^{2}\right)},
\end{aligned}
$$

where $\tau_{c}$ is the cumulative time constant which is defined as

$$
\frac{1}{\tau_{c}}=\frac{1}{\tau_{Q}}+\frac{1}{\tau_{q}} .
$$

$\omega_{i j}$ represents the energy difference between the $i$ th level in the reactant surface with the $j$ th level in the product surface, and $\omega_{X}(t)$ accounts for the energy gap along the $X$ coordinate which is changing due to the solvation dynamics.

\section{METHOD OF SOLUTION}

When the system is excited onto one of the vibronic level, $m$ of the reactant surface, that is, onto $V_{R, m}$, it is necessary to consider the electron transfer reactive sites (sinks) that are present along the intersections of the $V_{R, m}$ surface with the product surfaces, $V_{P, n}$. In addition, since populations can vibrationally relax to the lower levels from 
the initial $m$ surface, sinks present all along the intersection of the lower level surfaces with the $n$ surfaces also need to be considered. The resultant equation of motion is rather complicated.

Numerical solution of Eq. (3) for a two surface reaction with multiple broad reaction windows and with biphasic solvation dynamics with widely separated time scales, poses a formidable problem. We have solved this equation by using the Green's function technique ${ }^{19,55,61-64}$ as this has proven to be the most stable route, particularly for wide and variable strength sinks. Under the Green's function technique, the solution for Eq. (4) in the Laplace plane $P_{R, i}(X, z)$ is given by the following expression: ${ }^{19,55}$

$$
\begin{aligned}
P_{R, i}\left(X, z / X_{0}\right)= & \int d X^{\prime} G_{R, i}\left(X, z / X^{\prime}\right)\left\{P_{R, i}\left(X^{\prime}, t=0\right)-(1\right. \\
& +n) S(X) P_{n}\left(X^{\prime}, z\right)+S(X) P_{R, i}\left(X^{\prime}, z\right) \\
& \left.+k_{\mathrm{VER}} P_{R, i+1}\left(X^{\prime}, z\right)-k_{\mathrm{VER}} P_{R, i}\left(X^{\prime}, z\right)\right\},
\end{aligned}
$$

where $z$ is the Laplace frequency. A similar solution for the product surface $P_{P, i}$ can also be obtained and the coupled equations are used along with the initial excitation and the sink transfer conditions to obtain the survival probabilities on the reactant and the product individual vibronic states. The initial excitation on the reactant PES at the vibrational level $m$ is characterized using a $\delta$-function source at $X_{m}$. This can be written mathematical as, $P_{R, m}(X, t=0)=\delta(X$ $\left.-X_{m}\right) \delta_{i m}$. The expression for the Green's function in a harmonic surface is well-known and is given by

$$
\begin{aligned}
G\left(X, t / X^{\prime}, t=0\right)= & \frac{1}{\sqrt{2 \pi \sigma^{2}\left(1-S(t)^{2}\right)}} \\
& \times \exp \left\{-\frac{\left[X-X^{\prime} S(t)\right]^{2}}{2 \sigma^{2}\left[1-S(t)^{2}\right]}\right\},
\end{aligned}
$$

where $\sigma^{2}=k_{B} T / 2 \lambda_{X}$. A detailed derivation of survival probabilities starting from the generalized diffusion Eq. (3) is provided in the Appendix.

As the system is excited on to the $m$ th vibronic level in the reactant surface, $V_{R, m}$, it is necesary to consider the electron transfer reactive sites (sinks) that are present along the intersections of $V_{R, m}$ surface with the product surfaces. In addition since the population can vibrationally relax to the lower levels from the $m$ surface, sinks present all along the intersection of the lower levels with the product PES need to be considered. The sink function, $S(X)$ is assumed to be discretized and can be represented using the $\delta$-function as $S(X)=\sum_{s} k_{s}(X) \delta\left(X-X_{s}\right), k_{s}$ is the strength of each interval. The sink transfer rate, $k_{s}$ corresponding to the $m$ to $n$ transition involving the high frequency mode is $\left(2 \pi V_{\mathrm{el}}^{2} / \hbar\right)|\langle m \mid n\rangle|^{2}$, where $V_{\mathrm{el}}$ is the electronic coupling and $|\langle m \mid n\rangle|^{2}$ is the Franck-Condon overlap of the nuclear wave functions of the ground reactant, $m$ and the $n$th product states. The Franck-Condon factor between the $m$ and the $n$ state is given by the following relation

$$
\begin{aligned}
|\langle m \mid n\rangle|^{2}= & \exp \left(-\frac{\delta^{2}}{2}\right) m ! n ! \\
& \times\left[\sum_{r=0}^{\min (m, n)} \frac{(-1)^{m+n-r}(\delta / \sqrt{2})^{m+n-2 r}}{r !(m-r) !(n-r) !}\right]^{2},
\end{aligned}
$$

where $\delta^{2}=2 \lambda_{q} / h \nu_{q}$ is the coupling parameter. Substituting the sink function in Eq. (10) and performing the integration gives the expression for the population densities along the individual vibrational levels in the reactant and the product surfaces.

Our interest here is in the survival probability of the reactant, $P_{S}(t)$ which is obtained by summing over all $P_{R, i}(t)$. The latter is obtained from the following expression

$$
P_{R, i}(t)=\int d X \int d X_{0} P\left(X_{0}\right) P_{R, i}\left(X, t / X_{0}\right) .
$$

The Laplace transform of $P_{S}(t)$ is denoted by $P_{S}(z)$ $=\Sigma_{i} \int d X P(X, z)$. The method of obtaining the average electron transfer time $\left\langle\tau_{\text {et }}\right\rangle$ (Ref. 64) has been described in detail in our earlier works. ${ }^{55,56}$ The average electron transfer time $\left\langle\tau_{\text {et }}\right\rangle$ is related to the survival probability $P_{S}(t)$ through the following equation.

$$
\left\langle\tau_{\mathrm{et}}\right\rangle=\int_{0}^{\infty} d t P_{S}(t)=P_{S}(z=0) .
$$

\section{RESULTS AND DISCUSSION: ELECTRON TRANSFER KINETICS}

\section{A. Effect of reverse reaction within the Sumi-Marcus model}

The effect of the reverse electron transfer on the electron transfer dynamics is studied in the absence of VER. Figure 2 compares the time profile of the reactant survival probability for the case with and without the back electron transfer [only one reactant $(m=0)$ and one product surface $(n=0)$ have been considered here]. The values of the parameters are given in the figure caption. As seen from the figure, the reverse electron transfer slightly slows down the rate of electron transfer reaction in the intermediate to long time. Since the Sumi-Marcus model is also a two surface two state model (that is, without any high frequency vibrational modes), the results in Fig. 2 can be considered a generalization of the Sumi-Marcus model with back electron transfer. The reason that the recrossing in this particular model is not very significant is obviously due to the rapid relaxation and the removal of the population on the product side from the recrossing region. ${ }^{65,66}$ As discussed below, the reverse reaction can be more important under different circumstances.

When more than one product surfaces are involved in the electron transfer the excited population encounters more number of sinks and the survival probability decays quite rapidly at short times as shown in Fig. 3. However, the transferred population remains in their respective vibrational levels in the product surface (note that VER has not yet been considered) and since the free energy difference between the reactant and the product surfaces decreases with higher $n$, 


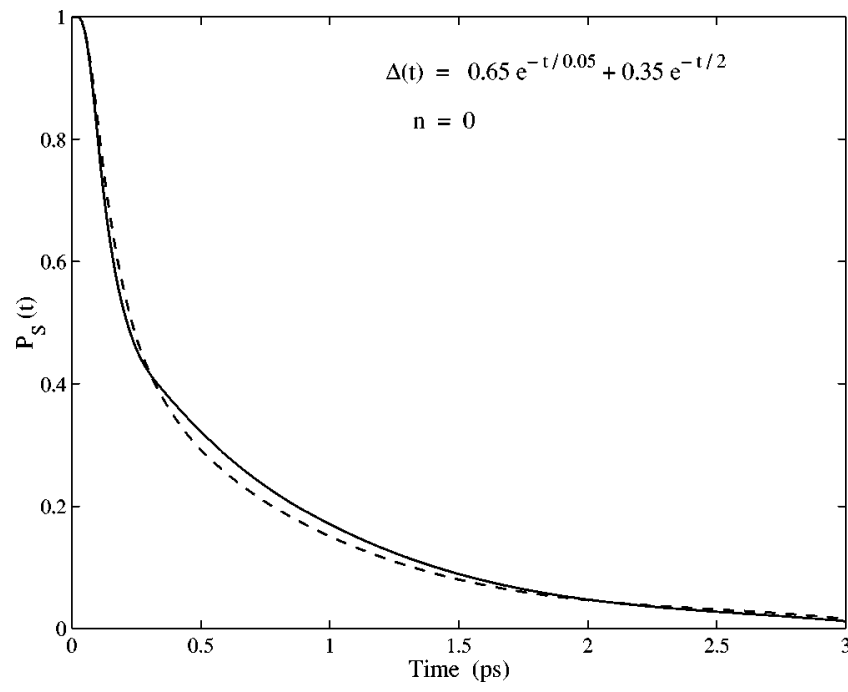

FIG. 2. The time-dependent survival probability on the reactant surface is plotted as a function of time (in ps) showing the nonexponential dynamics of the reactant population decay. In this graph, only one reactant and one product vibrational states are considered (that is, $m=n=0$ ) and the observed dynamics is for the following set of energy parameters (scaled by $1000 \mathrm{~cm}^{-1}: \lambda_{q}=1.5, \lambda_{X}=2.0, h \nu_{q}=1.0, \Delta G^{0}=-5.5$ and $\left.V_{\mathrm{el}}=0.9\right)$. The biexponential solvent time correlation function with $\tau_{E_{1}}=0.05, \tau_{E}=2.0, A$ $=0.65$, and $B=0.35$ are employed in the calculation. The solid line represents the case when "only" forward electron transfer is considered and the dotted line includes reverse or backward electron transfer as well. Times are scaled by picoseconds and all the calculations are performed at $298 \mathrm{~K}$.

recrossing of the transferred population is facilitated. Thus, in contrast to the expected behavior (i.e., an increase in the overall electron transfer rate due to the increase in the number of product surfaces), calculations without VER predict a complete reverse trend owing to the back electron transfer. This effect is severe and in fact a slight increase in the survival probability for the $n=2$ case is observed as shown in

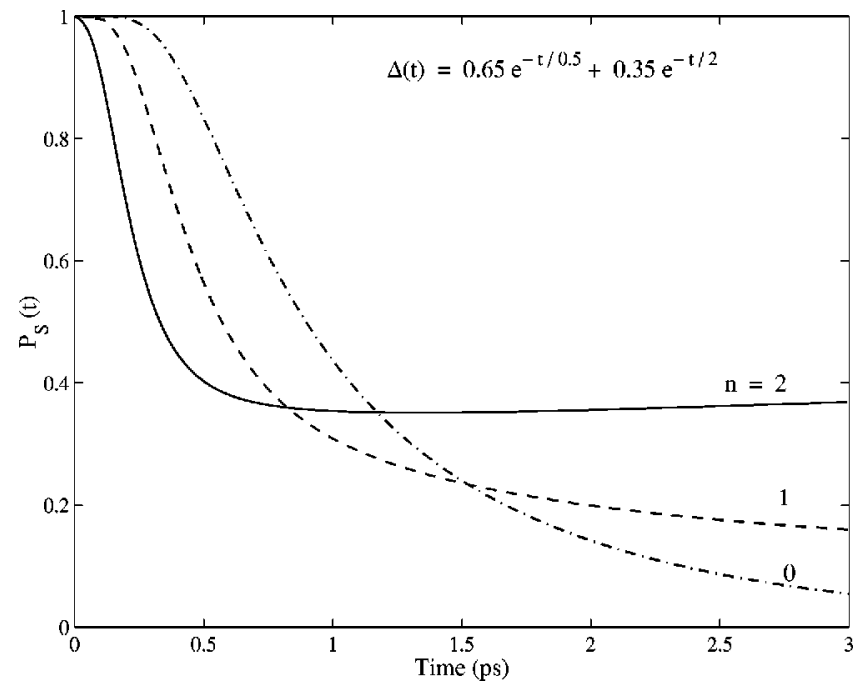

FIG. 3. The total reactant survival probability is plotted as a function of time (in ps) assuming a biexponential solvation time correlation function with the fast and the slow time constants, $\tau_{E_{1}}=0.5$ and $\tau_{E}=2.0$, respectively. The different curves represent participation of different number of product vibrational states, namely one $(n=0$, dashed-dotted line), two $(n=1$, dashed line), and three ( $n=2$, solid line) considered for a single reactant state ( $m$ $=0$ ). All the other parameters employed are the same as in Fig. 2.

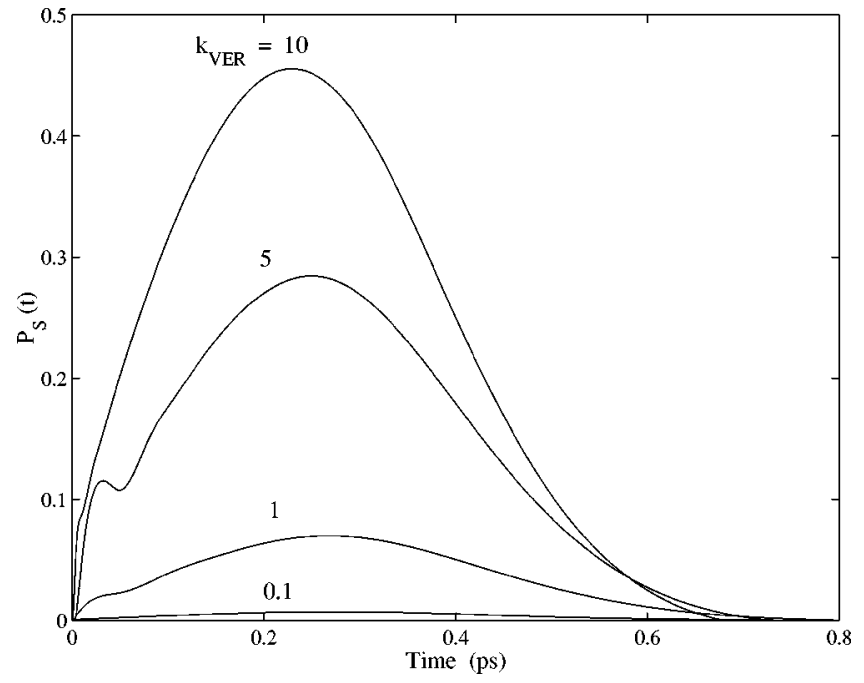

FIG. 4. The time (in ps) profile of the population decay of the ground vibronic state of the reactant surface (that is, $m=0$ ) for a system where the initial population is prepared in the $m=1$ vibrational state. The curves are obtained for varying vibrational energy relaxation rates, $k_{\mathrm{VER}}$ (marked on each graph). Calculations have been performed by including three vibrational product states. Equation (8) is employed for the solvation time correlation function with the time constant, $\tau_{G}=0.5 \mathrm{ps}$ and $\tau_{E}=2.0 \mathrm{ps}$. Free energy difference $\Delta G^{0}$ is chosen as $-5500 \mathrm{~cm}^{-1}$. All the other parameters remain the same as in Fig. 2.

Fig. 3. Thus VER play a nontrivial role in relaxing the transferred population in the product surface. In the following section we study the combined importance of VER and the reverse electron transfer.

\section{B. Importance of the VER in the hyybrid model}

The time evolution of population at any vibrational level is determined by the VER, the solvation dynamics and the electron transfer rate. The situation obviously is different for the ground $(n=0)$ vibrational state where only the last two are relevant. VER relaxes the population from the higher vibrational levels to the lower vibrational levels and the effect of VER on the reactant surface is to increase the ground vibrational state population and in the product surface it reduces the effect of recrossing dynamics.

Figure 4 depicts the effect of VER on the transient population of the zeroth vibrational level of the reactant $(m=0)$ when the initial excitation puts the probability on the $m=1$ level. When the VER rate is larger than the rate of electron transfer from the available sinks there is a sharp rise in the population at the $m=0$ level. Note that, this is a general result and not the consequence of the model employed. The reverse is true in the opposite limit. Surprisingly, however, this striking dependence of population distribution on the excited surface on the VER is not evident in the total survival probability, $P_{R}(t)$. The electron transfer time, when plotted against, $k_{\mathrm{VER}}$ remains essentially invariant. This is because electron transfer can occur either from $m=0$ or $m=1$ with nearly equal probability, for the given coupling strength. However, this scenario changes somewhat when $V_{\mathrm{el}}$ is small, like $100 \mathrm{~cm}^{-1}$. Then the rate of electron transfer registers a 


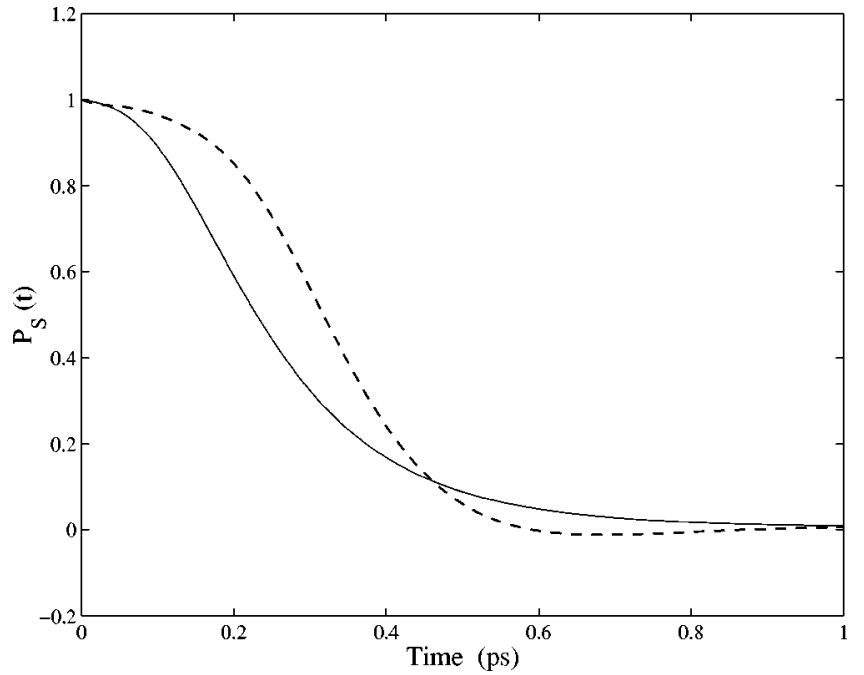

FIG. 5. The time (in ps) dependence of the total reactant survival probability calculated by involving 2 vibronic states of the reactant and 3 product vibrational states. The marked curves are obtained for the two different models of the solvation time correlation function, namely the biexponential model [Eq. (9)] with $\tau_{E_{1}}=0.5 \mathrm{ps}$ and $\tau_{E}=2.0 \mathrm{ps}$ (solid line) and the fast Gaussian-slow exponential model [Eq. (8)], with $\tau_{G}=0.5 \mathrm{ps}$ and $\tau_{E}$ $=2.0 \mathrm{ps}$ (dotted line). The vibrational energy relaxation rate is assumed as $\mathrm{ps}^{-1}$. Other parameters employed are the same as in Fig. 4.

small decrease with $k_{\mathrm{VER}}$. This is because solvation energy relaxation and $k_{\mathrm{VER}}$ populates regions where efficient sinks are absent.

\section{Importance of the Gaussian solvation dynamics}

Figure 5 shows the difference in the survival probability at the reactant surface when the ultrafast component of the solvation time correlation function is changed from the Gaussian to the exponential. The exponential $S(t)$ decreases the survival probability of the reactant surface rapidly at the short time and at longer times it decays steadily towards zero. In contrast, the Gaussian $S(t)$ shows a rather slow decay at the short time scale and predicts a fast decrease in the reactant population at the moderate to long time regime. Of course, the slower decay in the long time for the exponential case is due to larger average $\tau_{s}$ value for the latter. Theoretical studies show that even in this two surface multiple channel problem, the initial Gaussian component plays an important role. This is further depicted in Fig. 6, which demonstrates the sensitivity of the reaction to the magnitude of $\tau_{G}$.

It is interesting to note that the slow decay of the survival probability at the long time when the ultrafast solvation time constant is small. The possible reason for this is that the ultrafast component brings down the population faster along the PES and in that process encounters more number of sinks resulting in faster decrease in the survival probability at the transient time. The population that reaches the minimum of the reactant PES grows broader with time and covers the entire sink. This results in the slow decrease in the reactant population at longer time. This is further complicated by the reverse electron transfer. In contrast to the great sensitivity of the reaction to the ultrafast component, the reaction is much

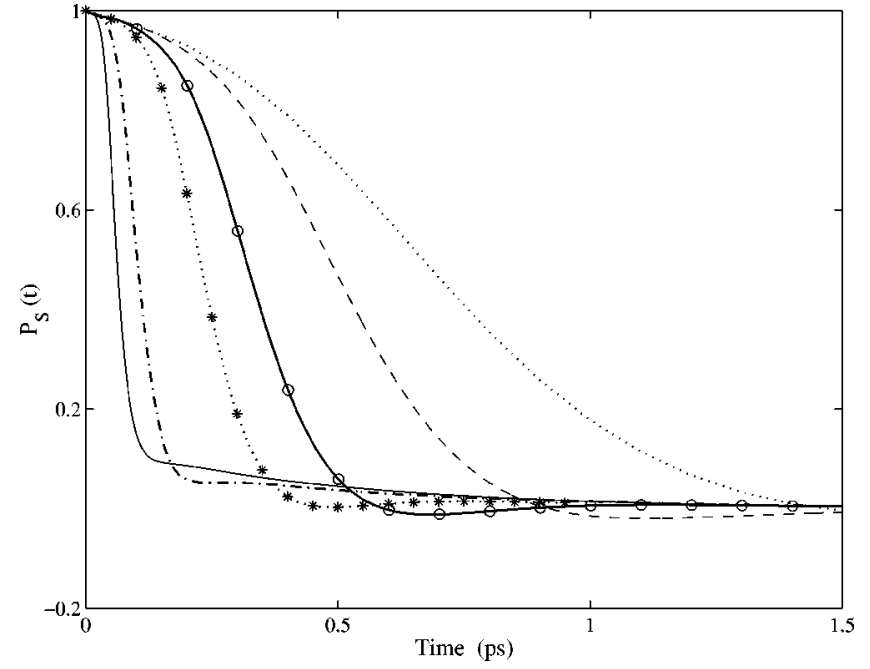

FIG. 6. The excited state reactant survival probability time (in ps) profile for different values of the fast component when the slow time constant, $\tau_{E}$ is kept fixed at $2.0 \mathrm{ps}$. The curves are obtained for $\tau_{G}$ values of $0.01,0.05,0.1$, $0.5,1.0$, and 2.0 (from left to right), all in ps. Remaining values are same as employed in Fig. 4.

less sensitive to $\tau_{E}$, as shown in Fig. 7. Figure 8(a) shows an insignificant variation in the $\left\langle\tau_{\mathrm{et}}\right\rangle$ when $\tau_{E}>\tau_{G}$. This feature clearly explains the control of the ultrafast solvation response on the decay of the excited state population at the short times. However, only when the value of $\tau_{E}$ is comparable or less than $\tau_{G}$ a significant change in the survival probability is seen at the short time. The decay of the survival probability at moderate and long time shows only a little variation for a large increase in the $\tau_{E}$ value, thus clearly predicting the decoupling of the electron transfer $d y$ namics from the slow solvent component.

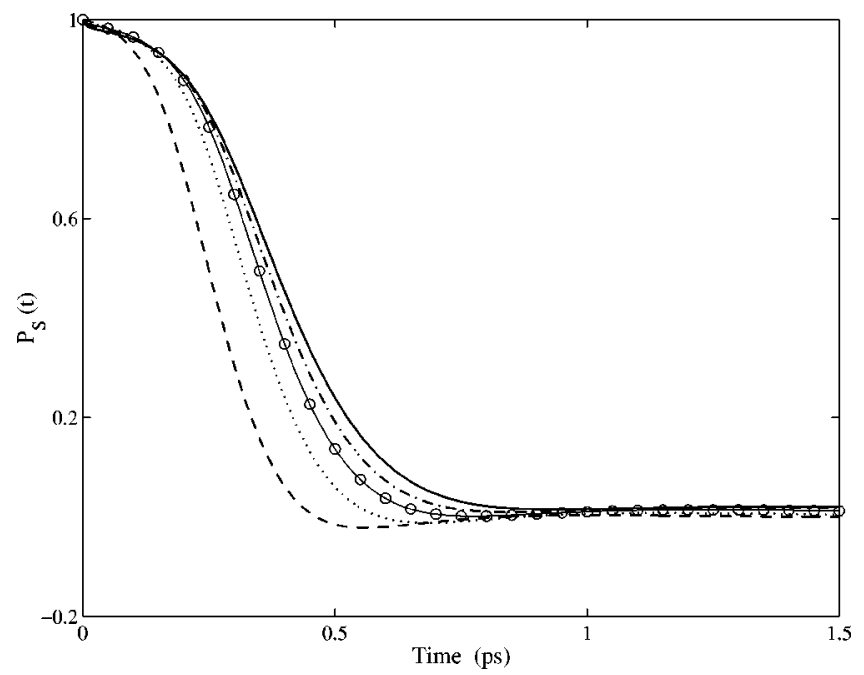

FIG. 7. The time (in ps) profile of the total reactant survival probability for several representative $\tau_{E}$ values [see Eq. (8)]. The value of $\tau_{G}$ is set at 0.5 ps. The curves from left to right are obtained for the $\tau_{E}$ values of $0.5,2.0$, 5.0, 10.0, and 20.0 (all in ps), respectively. Other parameters involved are the same as in Fig. 4. 

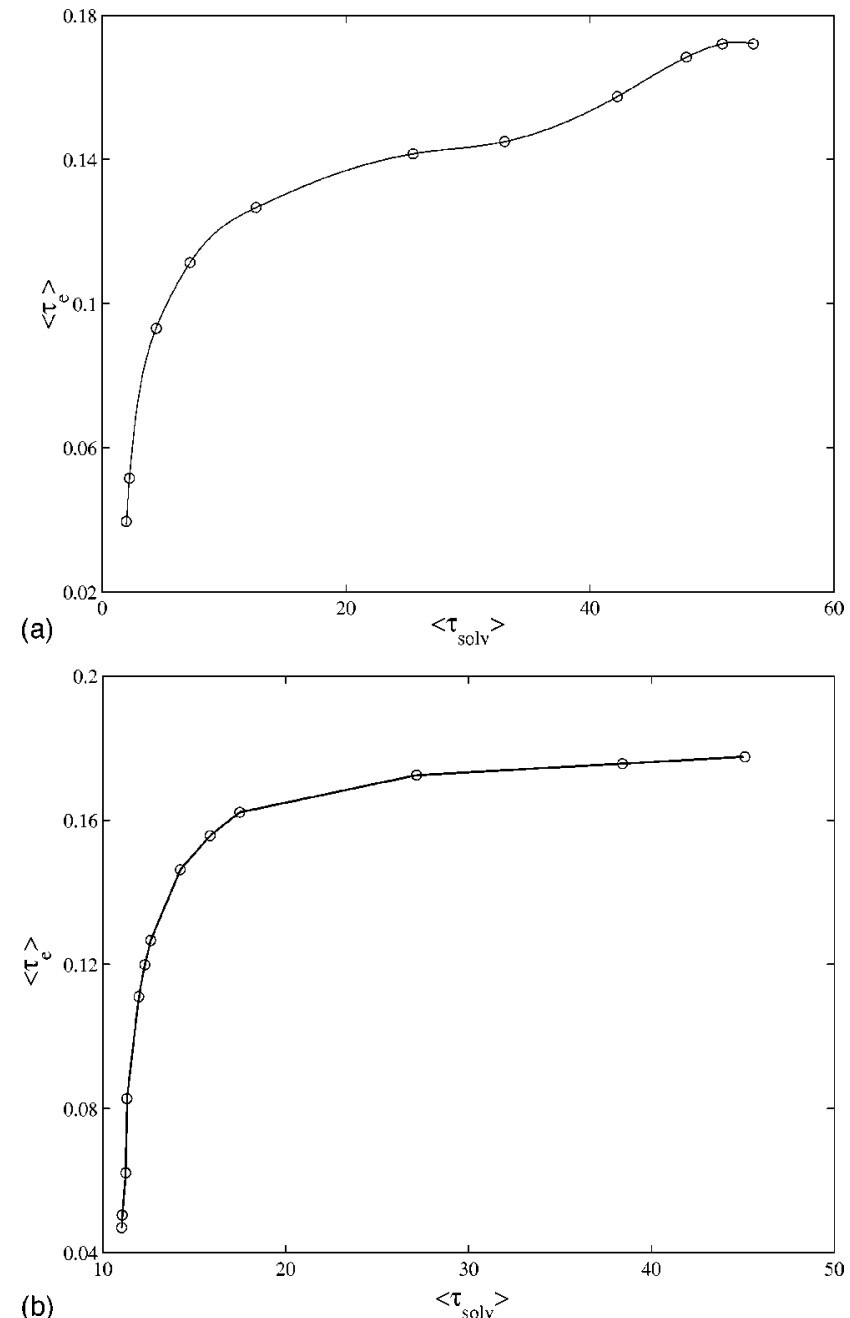

(b)

$<\tau_{\text {solv }}>$

FIG. 8. The calculated average electron transfer time (in ps) constant is plotted as a function of average solvation time constant which has been obtained from Eq. (6). (a) $\left\langle\tau_{s}\right\rangle$ is obtained by varying $\tau_{E}$ values by fixing $\tau_{G}$ as $0.5 \mathrm{ps}$. The curve shows a rapid increase when $\tau_{G}<\tau_{E}$ [see Eq. (8)] and when $\tau_{G} \geqslant \tau_{E}$ no appreciable change in electron transfer rate is observed. (b) $\left\langle\tau_{s}\right\rangle$ is calculated at a constant $\tau_{E}(=2.0 \mathrm{ps})$ value and by changing $\tau_{G}$. In these calculations, two reactant and four product vibrational surfaces are considered and the weight factors $A$ and $B$ in Eq. (8) are, respectively, 0.4 and 0.6. Other values remain the same as in Fig. 4.

\section{Effects of bimodal solvent response}

The effect of the ultrafast solvation rate on the electron transfer dynamics has already been depicted in Fig. 6. There is, however, another twist to the story of two time constants. In many systems, lowering of temperature can rapidly increase the value of the slow, exponential time constant (actually, this can also become nonexponential, but we shall not consider that aspect here). The first Gaussian time constant can show a weaker, even reverse, trend. Thus, the increase in the average solvation time comes from the slower decay. This can have an interesting effect on the average electron transfer rate, as depicted in Fig. 8. These figures show the decoupling of the electron transfer rate from the average solvation time, as was observed originally by Barbara et $a l^{26,27}$

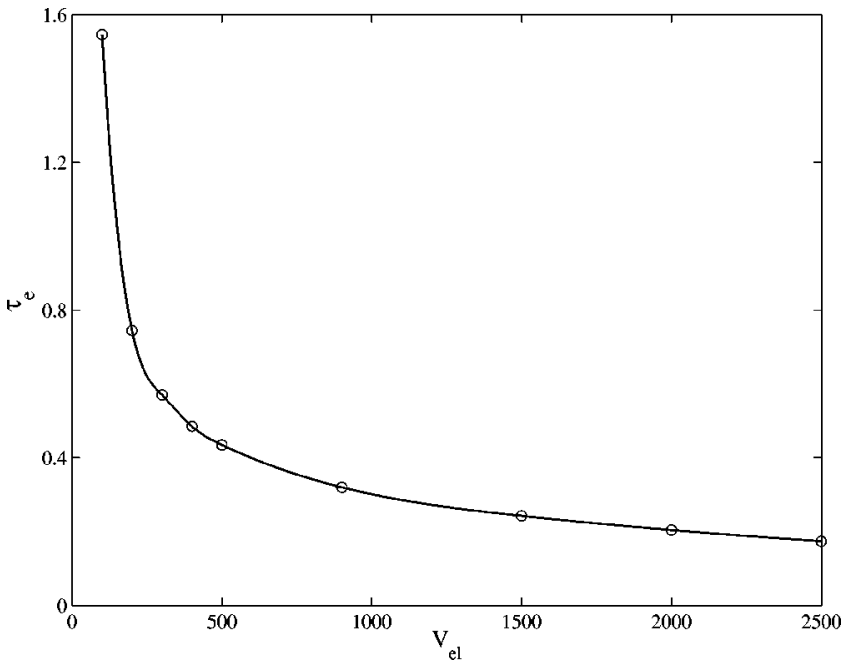

FIG. 9. The variation of average electron transfer time (in ps) constant with the electronic coupling constant, $V_{\mathrm{el}}\left(\mathrm{in} \mathrm{cm}^{-1}\right)$. Open circles are the data obtained and the solid line is the spline fit. All the other parameters remain the same as in Fig. 8.

\section{E. Sensitivity of the electron transfer rate to $V_{\mathrm{el}}$}

Figure 9 shows the effect of the electronic coupling factor on the rate of electron transfer reaction when $V_{\mathrm{el}}$ is varied from $50 \mathrm{~cm}^{-1}$ to $1000 \mathrm{~cm}^{-1}$. The electron transfer rate (which is inverse of the reaction time, $\tau_{e}$ ) increases linearly with $V_{\mathrm{el}}^{2}$ at low values of $V_{\mathrm{el}}^{2}$, as expected. At higher values of $V_{\mathrm{el}}$, a slower (sublinear) increase is observed.

During the photo-induced electron transfer there are two main processes that take place at the locally excited surface namely, the relaxation of the excited state population and the sink transfer to the product surface. In the limit of low $V_{\mathrm{el}}$ and relatively fast solvent relaxation, the rate is governed by the strength of the sink and the rate is proportional to $V_{\mathrm{el}}^{2}$. This limit is sometimes referred to as the nonadiabatic limit. ${ }^{8}$ However, when the sink is highly efficient, i.e., for large $V_{\mathrm{el}}$, the rate can be controlled by solvent relaxation and a weaker electron transfer rate dependence is envisaged. This limit is sometimes referred to as solvent relaxation limited regime (or even the adiabatic limit). In this regime, the rate can even show a fractional dependence on $V_{\mathrm{el}}^{2}$. These limits have been discussed at length in the literature. $6,7,9,67-69$

Figure 9, however, shows that even for large $V_{\mathrm{el}}$, the dependence on $V_{\mathrm{el}}$ does not become as weak as predicted in the Zusman or Sumi-Marcus or Rips-Jortner models. The reason can be understood following the logic of Jortner and Bixon. Even when solvent relaxation is slow, the reaction can proceed because a near-by sink is always available because of the reaction channels provided by the high frequency quantum mode (HFQM). However, one HFQM (employed here) might not be enough. On the other hand, these reaction channels are further broadened by the low frequency classical vibrational mode (the $Q$ mode). It is really interesting how the hybrid model captures such diverse behavior with relative ease. 

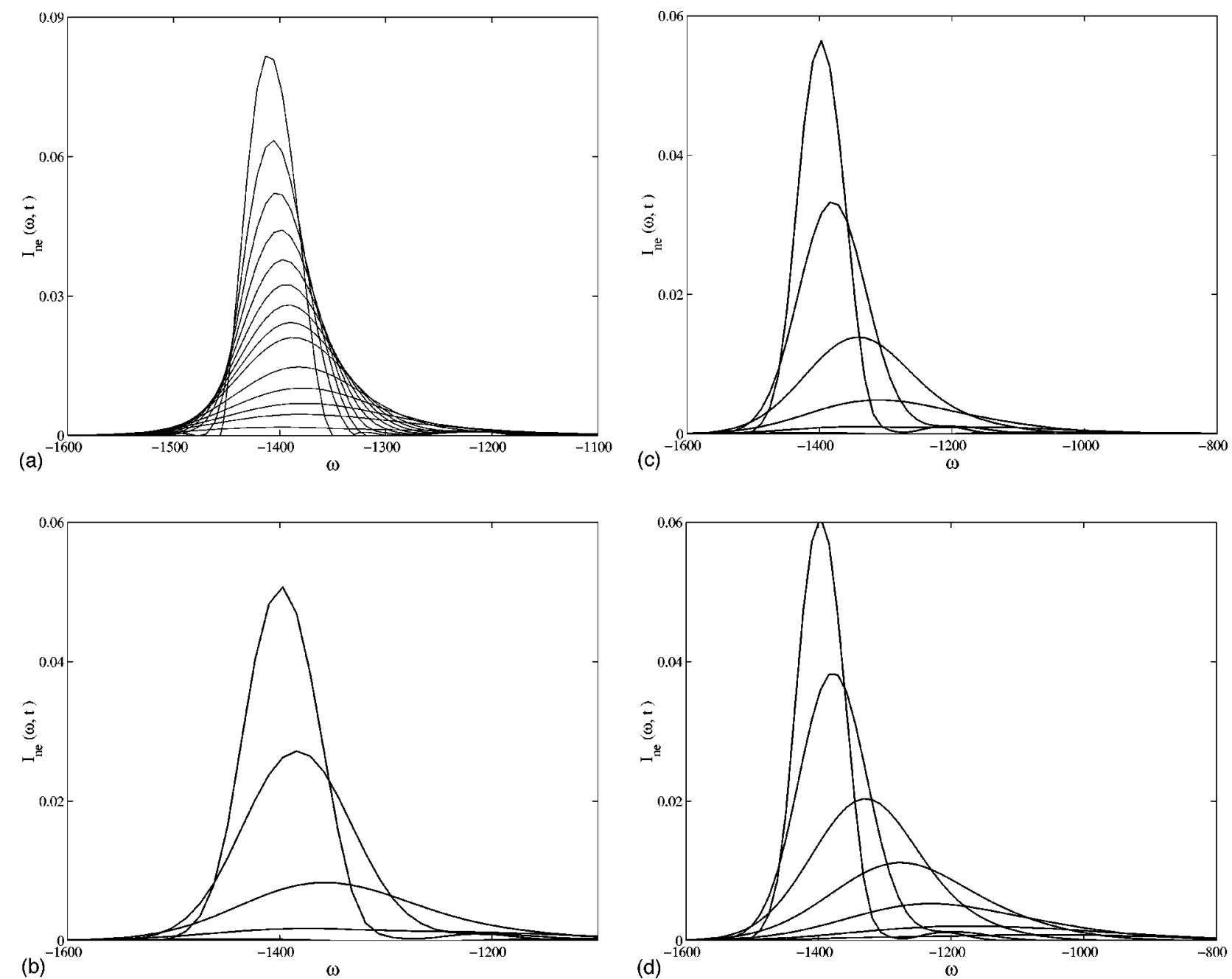

FIG. 10. Transient emission line shape, $I_{n e}(\omega, t)$, has been plotted at several representative times (in ps, indicated over the curve) for different electronic coupling constant $V_{\text {el }}$ values of (a) 0.9 , (b) 0.7 , (c) 0.5 and (d) 0.3 (all in $1000 \mathrm{~cm}^{-1}$ ). The plots show a slower and broader decay at low $V_{\text {el }}$ values. Other necessary values are the same as in Fig. 8 .

\section{ANALYSIS OF EMISSION LINE SHAPE DURING ELECTRON TRANSFER}

The nonequilibrium emission line shape in the presence of electron transfer has been analyzed to study the effects of electronic coupling and vibrational energy relaxation on the transient line shape. Figures 10(a)-10(d) show the timedependent line shapes for different electronic coupling values. The time origins are marked on the figures for convenience. These times are in ps, that is, the topmost curve in Fig. 10(a) is at $20 \mathrm{fs}$. The frequency is in $\mathrm{cm}^{-1}$. As the time progresses the line shape slowly broadens and the peak shifts towards the more positive $\omega$ values. When the coupling between the ground and the excited states is strong [Fig. 10(a)] the lineshape decays very rapidly and the spectra appears very broad at $0.25 \mathrm{ps}$ (note that $V_{\mathrm{el}}=900 \mathrm{~cm}^{-1}$ ). This is due to the stronger sink strength which aids the fast population decay from the excited surface. When the coupling between the electronic states are relatively weak the shift in the peak is clearly visible and the decay in the line shape is also considerable slow. Thus lowering the coupling strength broadens the line shape and slows the decay.

The rate of vibrational energy relaxation has interesting influence on the shape of the fluorescence spectra. At the stronger electronic coupling limit like say $900 \mathrm{~cm}^{-1}$, the effect of vibrational energy transfer is practically impossible to observe, due to the faster emission from the higher electronic state to the lower electronic states. Thus, at this condition, the relaxation along the vibrational coordinates are difficult to observe in the spectrum. Does it mean it is possible to observe the vibrational relaxation only in the low electronic coupling limit? The transient line shape observed for $V_{\mathrm{el}}$ $=100 \mathrm{~cm}^{-1}$ shows this interesting behavior. Figure 11 shows the emission line shapes for different $k_{\mathrm{VER}}$ values. An increase in the vibrational energy transfer rate accelerates the population decay from the initial (higher) vibrational level in the excited electronic states to the lower vibrational level. Thus, the emission from both the higher and the lower vibrational levels are recorded in the spectra and appears as two distinct peaks. However, this can be observed only at the short times, between 50 and 200 fs [Figs. 11(b) and 11(c)]. When the vibrational energy relaxation is slow the emission spectra doesn't produce this additional peak though the electronic coupling is less [Fig. 11 (a)]. This clearly demonstrates that the different peaks observed are due to the emission 

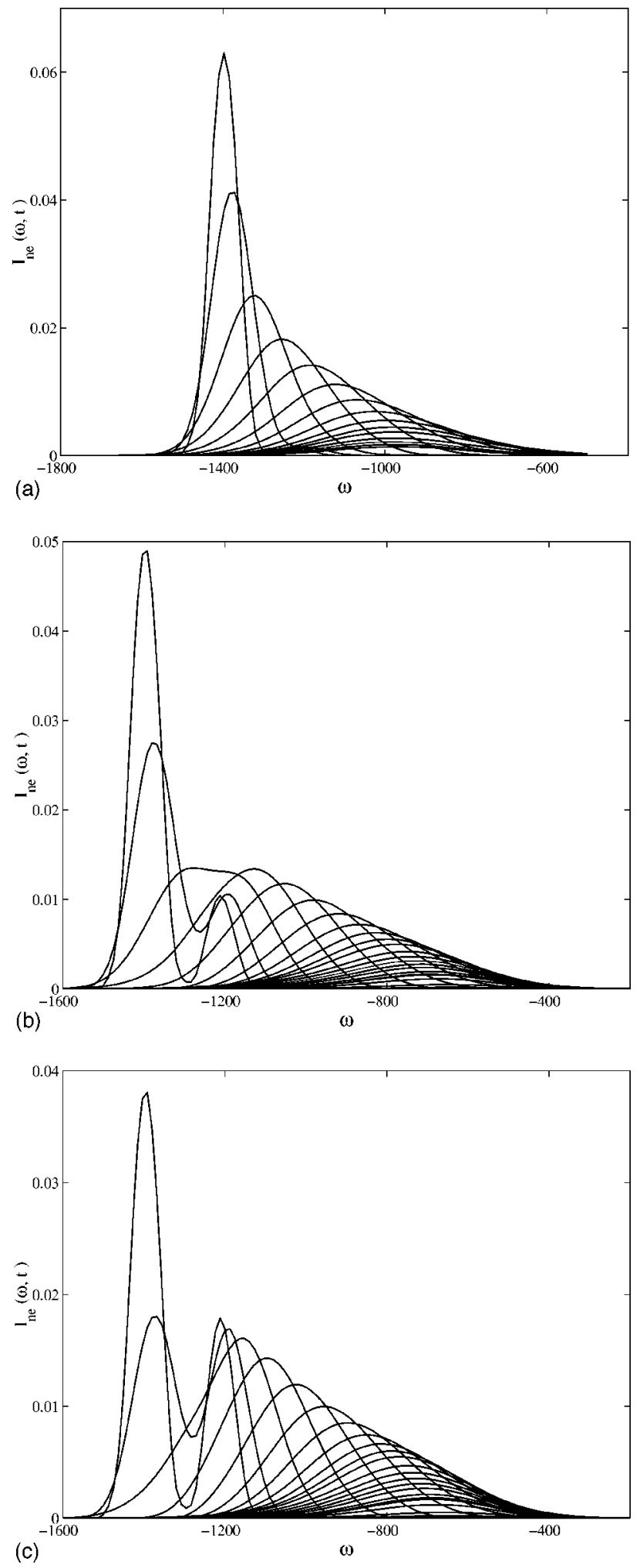

FIG. 11. Transient emission line shapes, $I_{n e}(\omega, t)$, have been plotted at several representative time (in ps) origins as indicated over the curve. Plots (a), (b) and (c) are obtained for different vibrational energy relaxation rates of 1,10 , and 20 (all in $\mathrm{ps}^{-1}$ ), respectively. A low electronic coupling constant value of $100 \mathrm{~cm}^{-1}$ is employed in these calculations. All the additional parameters needed remain the same as in Fig. 8.

from different vibrational states in the excited energy surface. At longer times, however, both these peaks merge and decays further in intensity with time. This state resolved spectra induced by fast vibrational energy transfer and low electronic coupling is certainly worth further investigation, particularly by experiments.

In the photo-induced electron transfer reactions, the initially populated states might be the vibrationally hot states of the reactant. When the reaction is deeply in the inverted regime, a reaction can occur from the nonequilibrium probability distribution on the reactant surface, that is, most of the population never gets time to equilibrate near the minimum of the reactant surface. Thus, the fluorescence spectrum decays while becoming red-shifted. For fast electron transfer, the relaxation of the high frequency vibrational mode might not be much faster than the electron transfer itself. In such a situation, the transient fluorescence spectrum shall contain the signature of the VER, as also of solvation dynamics. There is one more factor that will contribute to the line shape which is the existence of reverse electron transfer from the product to the reactant surface. This reverse transition give rise to one more line broadening mechanism and its effect has not been considered previously. The effect of solvation on the transient line shape involving electron transfer can also be studied using our present formalism.

\section{CONCLUSION}

We presented a theoretical formulation which can describe both the effects of vibrational energy relaxation and the reverse electron transfer on the kinetics of electron transfer reactions in solution. The new formulation, therefore, removes these limitations from the hybrid model.

Detailed numerical solution has been obtained for systems with model parameter values. Some of the results obtained are potentially important. For example, the relative insensitivity of the average electron transfer rate to the value of the vibrational energy relaxation rate for not too small values of the electronic coupling strength is significant. This means that as for the rate alone is concerned the controversy regarding the role of the VER can be mute. However, the details of population distribution in the reactant and product surfaces indeed depend on the magnitude of $k_{\mathrm{VER}}$ rather strongly. The situation can be different for small $V_{\mathrm{el}}$.

Another interesting result is the strong dependence of the rate on the value of the initial Gaussian component. Note that all earlier studies considered only exponential relaxation functions. The marked difference between the population decay profile for the Gaussian and exponential solvation correlation function also deserves special mention.

We also presented theoretical calculation of the transient fluorescence line shape from a photoexcited molecule undergoing an electron transfer reaction. As in many experimental situations, the system is in nonequilibrium state during fluorescence, undergoing simultaneous vibrational and solvation energy relaxations, both of which gives rise to a red-shift of the spectrum. A formulation has been developed by extending the well-known hybrid model to include not only VER and solvation dynamics but also reverse electron transfer. The reaction system is modeled by two surface, multiple, broad, reaction windows. The solvation dynamics is nonexponential, including an ultrafast Gaussian component. The new theory can describe the time dependent vibrational 
population distribution in the reactant and the product surfaces, during solvation dynamics and electron transfer. We have calculated the frequency resolved transient emission spectrum by using Franck-Condon rule from the nonequilibrium density of states.

The theoretical studies show that vibrational state resolved spectroscopy, both on the ground and excited state can reveal important information on the dynamics of electron transfer and on the role of vibrational dynamics in ETR. The effects of VER is particularly evident in the limit of small electronic coupling and fast vibrational energy relaxation.

\section{ACKNOWLEDGMENTS}

The authors thank Professor Abraham Nitzan for several interesting comments in the early stage of the work which partly motivated this work. It is a pleasure to thank Dr. N. Gayathri for helping us with the sink transfer rate calculation. Thanks are also due to Mr. G. Srinivas and Mr. R. K. Murarka for their help during various stages of this work. The work reported here was supported in parts from the grant received from the Department of Science and Technology, India, and from the Council of Scientific and Industrial Research.

\section{APPENDIX}

Here we present the mathematical details of the formalism for the multisurface, multistate electron transfer reaction with delocalized sink, reverse electron transfer, and vibrational relaxation. As can be anticipated, the formalism is a bit complicated.

\section{The coupled equation of motion}

The population variation in the space and the time coordinate at the ground vibrational surface $(i=0)$ of the reactant is given by

$$
\begin{aligned}
\frac{\partial P_{R, 0}(X, t)}{\partial t}= & \mathcal{L}_{R, 0} P_{R, 0}(X, t)-(1+n) S(X) P_{R, 0}(X, t) \\
& +S(X) P_{P}(X, t)+k_{\mathrm{VER}} P_{R, 1}(X, t),
\end{aligned}
$$

where $R$ and $P$ refers to the reactant and product PES, respectively, and $n$ represents the number of vibrational levels considered in the product PES. $S(X)$ is the positiondependent sink function, which describes the path along which the electron transfer takes place between the locally excited and charge transfer surfaces. The $\mathcal{L}_{R, 0}$ term simulates diffusion in a potential well $V_{R, 0}$. Let us assume $m$ be the initial vibrational state at which the population is prepared by photo-excitation. The time-evolution equation can be written as

$$
\begin{aligned}
\frac{\partial P_{R, m}(X, t)}{\partial t}= & \mathcal{L}_{R, m} P_{R, m}(X, t)-(1+n) S(X) P_{R, m}(X, t) \\
& +S(X) P_{P}(X, t)-k_{\mathrm{VER}} P_{R, m}(X, t), \quad
\end{aligned}
$$

and for any other vibrational level $i$ in between 0 and the $m$ th level in the reactant PES, population decay is given by the equation,

$$
\begin{aligned}
\frac{\partial P_{R, i}(X, t)}{\partial t}= & \mathcal{L}_{R, i} P_{R, i}(X, t)-(1+n) S(X) P_{R, i}(X, t) \\
& +S(X) P_{P}(X, t)+k_{\mathrm{VER}} P_{R, i+1}(X, t) \\
& -k_{\mathrm{VER}} P_{R, i}(X, t) .
\end{aligned}
$$

The modified Smoluchowski equation for the diffusion motion along the ground vibrational surface in the product surface can be expressed as,

$$
\begin{aligned}
\frac{\partial P_{P, 0}(\hat{X}, t)}{\partial t}= & \mathcal{L}_{P, 0} P_{P, 0}(\hat{X}, t)-(1+m) S(\hat{X}) P_{P, 0}(\hat{X}, t) \\
& +S(\hat{X}) P_{R}(\hat{X}, t)+k_{\mathrm{VER}} P_{P, 1}(\hat{X}, t),
\end{aligned}
$$

where $\hat{X}=1-X$ and $P$ indicates that the diffusive motion is along the product PES. An equation for the $n$th vibrational state can be written as

$$
\begin{aligned}
\frac{\partial P_{P, n}(\hat{X}, t)}{\partial t}= & \mathcal{L}_{P, n} P_{P, n}(\hat{X}, t)-(1+m) S(\hat{X}) P_{P, n}(\hat{X}, t) \\
& +S(\hat{X}) P_{R}(\hat{X}, t)-k_{\mathrm{VER}} P_{P, n}(\hat{X}, t) .
\end{aligned}
$$

For the vibrational level(s) $j$ between 0 and the $n$th level, population decay is given by the expression

$$
\begin{aligned}
\frac{\partial P_{P, j}(\hat{X}, t)}{\partial t}= & \mathcal{L}_{R, j} P_{P, j}(\hat{X}, t)-(1+m) S(\hat{X}) P_{R, j}(\hat{X}, t) \\
& +S(\hat{X}) P_{P}(\hat{X}, t)+k_{\mathrm{VER}} P_{R, j+1}(\hat{X}, t) \\
& -k_{\mathrm{VER}} P_{R, i}(\hat{X}, t),
\end{aligned}
$$

where $P_{R}\left(P_{P}\right)$ indicates the transfer from the reactant (product) to all the product (reactant) vibrational levels.

Laplace transforming the dynamical equations for the reactant surfaces leads to the following equations:

$$
\begin{aligned}
& {\left[z-\mathcal{L}_{R, 0}\right] P_{R, 0}(X, z)} \\
& =P_{R, 0}(X, t=0)-(1+n) S(X) P_{R, 0}(X, z)+S(X) P_{n}(X, z) \\
& +k_{\mathrm{VER}} P_{R, 1}(X, z) \text {, } \\
& {\left[z-\mathcal{L}_{R, i}\right] P_{R, i}(X, z)} \\
& =P_{R, i}(X, t=0)-(1+n) S(X) P_{R, i}(X, z)+S(X) P_{n}(X, z) \\
& -k_{\mathrm{VER}} P_{R, i}(X, z)+k_{\mathrm{VER}} P_{R, i+1}(X, z), \\
& {\left[z-\mathcal{L}_{R, m}\right] P_{R, m}(X, z)} \\
& =P_{R, m}(X, t=0)-(1+n) S(X) P_{R, m}(X, z) \\
& +S(X) P_{n}(X, z)-k_{\mathrm{VER}} P_{R, m}(X, z),
\end{aligned}
$$

where $P_{R, i}(X, t=0)$ denotes the initial equilibrium probability distribution on the $i$ th vibrational level in the reactant PES and $z$ is the Laplace frequency conjugate to the time $t$. Similar ones are obtained for the product surfaces. 


\section{The solution by Green's function technique}

The above Eqs. (A7)-(A9) can be solved using Green's function technique. By definition, the Green's function for both the reactant and the product surfaces incorporating all the vibrational states follows the equations

$$
\begin{aligned}
& {\left[z-\mathcal{L}_{R, 0}\right] G_{R, 0}\left(X, z / X^{\prime}\right)=\delta\left(X-X^{\prime}\right),} \\
& {\left[z-\mathcal{L}_{R, i}\right] G_{R, i}\left(X, z / X^{\prime}\right)=\delta\left(X-X^{\prime}\right),} \\
& {\left[z-\mathcal{L}_{R, m}\right] G_{R, m}\left(X, z / X^{\prime}\right)=\delta\left(X-X^{\prime}\right),} \\
& {\left[z-\mathcal{L}_{P, 0}\right] G_{P, 0}\left(\hat{X}, z / \hat{X}^{\prime}\right)=\delta\left(\hat{X}-\hat{X}^{\prime}\right),} \\
& {\left[z-\mathcal{L}_{P, j}\right] G_{P, j}\left(\hat{X}, z / \hat{X}^{\prime}\right)=\delta\left(\hat{X}-\hat{X}^{\prime}\right),} \\
& {\left[z-\mathcal{L}_{P, n}\right] G_{P, n}\left(\hat{X}, z / \hat{X}^{\prime}\right)=\delta\left(\hat{X}-\hat{X}^{\prime}\right) .}
\end{aligned}
$$

The solutions for $P_{R}$ and $P_{P}$ are given in terms of the following coupled equations:

$$
\begin{aligned}
P_{R, 0}(X, z)= & \int d X^{\prime} G_{R, 0}\left(X, z / X^{\prime}\right)\left\{P_{R, 0}\left(X^{\prime}, t=0\right)-(1\right. \\
& +n) S\left(X^{\prime}\right) P_{n}\left(X^{\prime}, z\right)+S\left(X^{\prime}\right) P_{R, 0}\left(X^{\prime}, z\right) \\
& \left.+k_{\mathrm{VER}} P_{R, 1}\left(X^{\prime}, z\right)\right\}, \\
P_{R, i}(X, z)= & \int d X^{\prime} G_{R, i}\left(X, z / X^{\prime}\right)\left\{P_{R, i}\left(X^{\prime}, t=0\right)-(1\right. \\
& +n) S\left(X^{\prime}\right) P_{n}\left(X^{\prime}, z\right)+S\left(X^{\prime}\right) P_{R, i}\left(X^{\prime}, z\right) \\
& \left.+k_{\mathrm{VER}} P_{R, i+1}\left(X^{\prime}, z\right)-k_{\mathrm{VER}} P_{R, i}\left(X^{\prime}, z\right)\right\}, \\
P_{R, m}(X, z)= & \int d X^{\prime} G_{R, m}\left(X, z / X^{\prime}\right)\left\{P_{R, m}\left(X^{\prime}, t=0\right)-(1\right. \\
& +n) S\left(X^{\prime}\right) P_{n}\left(X^{\prime}, z\right)+S\left(X^{\prime}\right) P_{R, m}\left(X^{\prime}, z\right) \\
& \left.-k_{\mathrm{VER}} P_{R, m}\left(X^{\prime}, z\right)\right\} .
\end{aligned}
$$

Similar equations for the product surfaces can be represented as

$$
\begin{aligned}
P_{P, 0}(\hat{X}, z)= & \int d \hat{X}^{\prime} G_{P, 0}\left(\hat{X}, z / \hat{X}^{\prime}\right)\left\{P_{P, 0}\left(\hat{X}^{\prime}, t=0\right)-(1\right. \\
& +n) S\left(\hat{X}^{\prime}\right) P_{m}\left(\hat{X}^{\prime}, z\right)+S\left(\hat{X}^{\prime}\right) P_{P, 0}\left(\hat{X}^{\prime}, z\right) \\
& \left.+k_{\mathrm{VER}} P_{P, 1}\left(\hat{X}^{\prime}, z\right)\right\}, \\
P_{P, j}(\hat{X}, z)= & \int d \hat{X}^{\prime} G_{P, j}\left(\hat{X}, z / \hat{X}^{\prime}\right)\left\{P_{P, j}\left(\hat{X}^{\prime}, t=0\right)-(1\right. \\
& +n) S\left(\hat{X}^{\prime}\right) P_{m}\left(\hat{X}^{\prime}, z\right)+S\left(\hat{X}^{\prime}\right) P_{P, j}\left(\hat{X}^{\prime}, z\right) \\
& \left.+k_{\mathrm{VER}} P_{P, j+1}\left(\hat{X}^{\prime}, z\right)-k_{\mathrm{VER}} P_{P_{j}}\left(\hat{X}^{\prime}, z\right)\right\} \\
P_{P, n}(\hat{X}, z)= & \int d \hat{X}^{\prime} G_{P, n}\left(\hat{X}, z / \hat{X}^{\prime}\right)\left\{P_{P, n}\left(\hat{X}^{\prime}, t=0\right)-(1\right. \\
& +n) S\left(\hat{X}^{\prime}\right) P_{m}\left(\hat{X}^{\prime}, z\right)+S\left(\hat{X}^{\prime}\right) P_{P, n}\left(\hat{X}^{\prime}, z\right) \\
& \left.-k_{\mathrm{VER}} P_{P, n}\left(\hat{X}^{\prime}, z\right)\right\} .
\end{aligned}
$$

The initial population that is excited on the reactant (locally excited) surface may be characterized as a deltafunction source at $X_{0}$ on the $m$ th high frequency vibrational state. This condition can be stated mathematically as $P_{R, m}(X, t=0)=\delta\left(X-X_{m}\right) \delta_{i m}$. Here we also assume the populations in the higher vibrational states and in the product potential energy surface are zero, i.e., $P_{R, i}(X, t=0)=0$; $P_{R, m}(X, t=0)=0 ; \quad P_{P, 0}(\hat{X}, t=0)=0 ; \quad P_{P, j}(\hat{X}, t=0)=0 ;$ $P_{P, n}(\hat{X}, t=0)=0$.

\section{Discretization of the sink function}

The sink function, $S(X)$ can be written as $S(X)$ $=\int d X^{\prime} S\left(X^{\prime}\right) \delta\left(X-X^{\prime}\right)$. The property of dividing the continuous sink curve into a number of intervals is exploited to relate the sink function to the intrinsic sink rate, $k_{s}$,

$$
S\left(X^{\prime}\right)=\sum_{0} k_{s} \delta\left(X-X_{s}\right) .
$$

The use of sink function in Eqs. (A16)-(A21) and solving for the solution of $P_{R}$ and $P_{P}$ we obtain the following equations:

$$
\begin{aligned}
P_{R, 0}(X, z)= & G_{R, 0}\left(X, z / X_{0}\right)-(1+n) \\
& \times \sum_{s} k_{s}^{R, 0} G_{R, 0}\left(X, z / X_{s}\right) P_{R, 0}\left(X_{s}, z\right) \\
& +\sum_{s} k_{s}^{R, 0} G_{R, 0}\left(X, z / X_{s}\right) P_{P}\left(X_{s}, z\right) \\
& +k_{\mathrm{VER}} \int d X^{\prime} G_{R, 0}\left(X, z / X^{\prime}\right) P_{R, 1}\left(X^{\prime}, z\right), \\
P_{R, i}(X, z)= & G_{R, i}\left(X, z / X_{0}\right)-(1+n) \\
& \times \sum_{s} k_{s}^{R, 0} G_{R, i}\left(X, z / X_{s}\right) P_{R, i}\left(X_{s}, z\right) \\
& +\sum_{s} k_{s}^{R, i} G_{R, i}\left(X, z / X_{s}\right) P_{P}\left(X_{s}, z\right) \\
& +k_{\mathrm{VER}} \int d X^{\prime} G_{R, i}\left(X, z / X^{\prime}\right) P_{R, i+1}\left(X^{\prime}, z\right) \\
& -k_{\mathrm{VER}} \int d X^{\prime} G_{R, i}\left(X, z / X^{\prime}\right) P_{R, i}\left(X^{\prime}, z\right),
\end{aligned}
$$

$$
\begin{aligned}
P_{R, m}(X, z)= & G_{R, m}\left(X, z / X_{0}\right)-(1+n) \\
& \times \sum_{s} k_{s}^{R, m} G_{R, m}\left(X, z / X_{s}\right) P_{R, m}\left(X_{s}, z\right) \\
& +\sum_{s} k_{s}^{R, m} G_{R, m}\left(X, z / X_{s}\right) P_{P}\left(X_{s}, z\right) \\
& -k_{\mathrm{VER}} \int d X^{\prime} G_{R, m}\left(X, z / X^{\prime}\right) P_{R, m}\left(X^{\prime}, z\right),
\end{aligned}
$$


where $k_{s}^{R, 0}$ represents the sink transfer rate for the 0th vibrational state in the reactant surface with all the vibrational states in the product surface. Similar ones are for the product surface.

The integral in Eqs. (A23)-(A25) can be discretized to give,

$$
\begin{aligned}
& P_{R, 0}(X, z)=G_{R, 0}\left(X, z / X_{0}\right)-(1+n) \\
& \times \sum_{s} k_{s}^{R, 0} G_{R, 0}\left(X, z / X_{s}\right) P_{R, 0}\left(X_{s}, z\right) \\
& +\sum_{s} k_{s}^{R, 0} G_{R, 0}\left(X, z / X_{s}\right) P_{P}\left(X_{s}, z\right) \\
& +R \sum_{k=1}^{N} G_{R, 0}\left(X, z / X_{k}\right) P_{R, 1}\left(X_{k}, z\right), \\
& P_{R, i}(X, z)=G_{R, i}\left(X, z / X_{0}\right)-(1+n) \\
& \times \sum_{s} k_{s}^{R, 0} G_{R, i}\left(X, z / X_{s}\right) P_{R, i}\left(X_{s}, z\right) \\
& +\sum_{s} k_{s}^{R, i} G_{R, i}\left(X, z / X_{s}\right) P_{P}\left(X_{s}, z\right) \\
& +R \sum_{k=1}^{N} G_{R, i}\left(X, z / X_{k}\right) P_{R, i+1}\left(X_{k}, z\right) \\
& -R \sum_{k=1}^{N} G_{R, i}\left(X, z / X_{k}\right) P_{R, i}\left(X_{k}, z\right), \\
& P_{R, m}(X, z)=G_{R, m}\left(X, z / X_{0}\right)-(1+n) \\
& \times \sum_{s} k_{s}^{R, m} G_{R, m}\left(X, z / X_{s}\right) P_{R, m}\left(X_{s}, z\right) \\
& +\sum_{s} k_{s}^{R, m} G_{R, m}\left(X, z / X_{s}\right) P_{P}\left(X_{s}, z\right) \\
& -R \sum_{k=1}^{N} G_{R, m}\left(X, z / X_{k}\right) P_{R, m}\left(X_{k}, z\right),
\end{aligned}
$$

where $R=h k_{\mathrm{VER}}, h$ is the width of the integral, and $N$ is the number of integration points. Note that sink points are discretized and the integral in the VER term is also discretized. Thus in effect sink points will be embedded inside the discretized VER term and while computation proper care needs to be taken to incorporate the VER and sink effects. ${ }^{19}$ Similar ones are for the product surface.

\section{The matrix formulation}

A set of linear equations can be constructed from Eqs. (A26)-(A28) and corresponding equations from the product surface. The solution of this leads to $P_{R}$ and $P_{P}$. The linear equation can be written in a matrix form as

$$
\mathbf{B} \cdot \mathbf{P}=\mathbf{G}_{\mathbf{0}},
$$

where the elements of the $\mathbf{B}$ matrix contains information about the sink and VER and $\mathbf{G}_{\mathbf{0}}$ matrix contains the terms involved in the initial excited state and the initial population of the states, if any at all. In this problem, however, we consider that initially the vibrational states are empty and the population is induced only by the local excitation using a photon pulse.

\section{Survival probability}

Survival probabilities at the individual vibronic states along the reactant surface, $P_{S, u}(t)$ can be defined as

$$
P_{S, u}(t)=\int_{0}^{\infty} P_{R, u}(X, t)
$$

and the overall survival probability along the reactant surface, $P_{S}(t)$ can be written as a sum over all the vibrational states,

$$
P_{S}(t)=\sum_{u} P_{S, u}(t)
$$

where $u=0,1,2, \ldots, m$. Similarly one can also define a timedependent probability functions for the product surface as

$$
P_{S, v}(t)=\int_{0}^{\infty} P_{P, v}(X, t)
$$

and

$$
P_{S, P}(t)=\sum_{v} P_{S, v}(t)
$$

where $v=0,1,2 \ldots n$.

The population on the reactant surface as a function of the Laplace frequency can be written in the form,

$$
\begin{aligned}
P_{R, 0}(z)= & \frac{1}{z}\left\{-(1+n) \sum_{s} k_{s}^{R, 0} P_{R, 0}\left(X_{s}, z\right)\right. \\
& \left.+\sum_{s} k_{s}^{R, 0} P_{P}\left(X_{s}, z\right)+R \sum_{k=0}^{N} P_{R, 1}\left(X_{k}, z\right)\right\}, \\
P_{R, i}(z)= & \frac{1}{z}\left\{-(1+n) \sum_{s} k_{s}^{R, i} P_{R, i}\left(X_{s}, z\right)\right. \\
& +\sum_{s} k_{s}^{R, j} P_{P}\left(X_{s}, z\right)+R \sum_{k=0}^{N} P_{R, i+1}\left(X_{k}, z\right) \\
& \left.-R \sum_{k=0}^{N} P_{R, i}\left(X_{k}, z\right)\right\}, \\
& \left.+\sum_{s} k_{s}^{R, m} P_{P}\left(X_{s}, z\right)-R \sum_{k=0}^{N} P_{R, m}\left(X_{k}, z\right)\right\}, \\
P_{R, m}(z)= & \frac{1}{z}\left\{1-(1+n) \sum_{s} k_{s}^{R, m} P_{R, m}\left(X_{s}, z\right)\right. \\
& (\mathrm{A} 3)
\end{aligned}
$$




$$
\begin{aligned}
P_{P, 0}(z)= & \frac{1}{z}\left\{-(1+m) \sum_{s} k_{s}^{P, 0} P_{P, 0}\left(\hat{X}_{s}, z\right)\right. \\
& \left.+\sum_{s} k_{s}^{P, 0} P_{R}\left(\hat{X}_{s}, z\right)+R \sum_{k=0}^{N} P_{P, 1}\left(\hat{X}_{k}, z\right)\right\}, \\
P_{P, j}(z)= & \frac{1}{z}\left\{-(1+m) \sum_{s} k_{s}^{P, j} P_{P, j}\left(\hat{X}_{s}, z\right)\right. \\
& +\sum_{s} k_{s}^{P, j} P_{R}\left(\hat{X}_{s}, z\right)-R \sum_{k=0}^{N} P_{P, j}\left(\hat{X}_{k}, z\right) \\
& \left.+R \sum_{k=0}^{N} P_{P, j}+1\left(\hat{X}_{k}, z\right)\right\}, \\
& \left.+\sum_{s} k_{s}^{P, m} P_{R}\left(\hat{X}_{s}, z\right)-R \sum_{k=0}^{N} P_{P, m}\left(\hat{X}_{k}, z\right)\right\} .
\end{aligned}
$$

Note Eq. (A36) contains the information concerning the initial photoexcitation.

${ }^{1}$ R. A. Marcus, J. Chem. Phys. 24, 979 (1956).

${ }^{2}$ R. A. Marcus, Annu. Rev. Phys. Chem. 15, 155 (1964).

${ }^{3}$ R. A. Marcus, J. Chem. Phys. 43, 679 (1965).

${ }^{4}$ G. L. Closs and J. R. Miller, Science 240, 440 (1988).

${ }^{5}$ J. Jortner and M. Bixon, Adv. Chem. Phys., Vol. 106, Parts 1 and 2: Electron Transfer From Isolated Molecules to Biomolecules (Wiley, New York, 1999), and references therein.

${ }^{6}$ L. D. Zusman, Chem. Phys. 49, 295 (1980); 119, 51 (1988).

${ }^{7}$ T. Fonseca, J. Chem. Phys. 91, 2869 (1989); T. Fonseca, Chem. Phys. Lett. 162, 491 (1989).

${ }^{8}$ J. T. Hynes, J. Phys. Chem. 90, 3701 (1986).

${ }^{9}$ H. Sumi and R. A. Marcus, J. Chem. Phys. 84, 4894 (1986).

${ }^{10} \mathrm{~J}$. Ulstrup, Charge Transfer Processes in Condensed Media, Lecture Notes in Chemistry (Springer, New York, 1979), and references therein.

${ }^{11}$ M. D. Newton, Int. J. Quantum Chem., Symp. 14, 363 (1980).

${ }^{12}$ M. D. Newton, ACS Symp. Ser. 64, 255 (1982).

${ }^{13}$ M. D. Newton, Chem. Rev. 91, 767 (1991).

${ }^{14}$ N. Sutin, Prog. Inorg. Chem. 30, 441 (1983).

${ }^{15}$ A. Warshel and W. W. Parson, Annu. Rev. Phys. Chem. 42, 279 (1991).

${ }^{16}$ H. Heitele, Angew. Chem. Int. Ed. Engl. 32, 359 (1993).

${ }^{17}$ P. Chen and T. J. Meyer, Chem. Rev. 98, 1439 (1998).

${ }^{18}$ G. Iversen, E. P. Friis, Y. Kharkats, A. M. Kuznetsov, and J. Ulstrup, J. Bio. Inorg. Chem. 3, 229 (1998).

${ }^{19}$ B. Bagchi and N. Gayathri, Adv. Chem. Phys. 107, 1 (1999).

${ }^{20}$ D. Rehm and A. Weller, Isr. J. Chem. 8, 259 (1970).

${ }^{21}$ M. Tachiya and S. Murata, J. Am. Chem. Soc. 116, 2424 (1994).

${ }^{22}$ S. Murata and M. Tachiya, J. Phys. Chem. 100, 4064 (1996).

${ }^{23}$ C. P. Andrieux, J. Savient, A. Tallec, R. Tardivel, and C. Tardy, J. Am. Chem. Soc. 118, 9788 (1996).

${ }^{24}$ A. E. Johnson, N. E. Levinger, W. Jarzeba, R. E. Schleif, D. A. V. Kliner, and P. F. Barbara, Chem. Phys. 176, 555 (1993).

${ }^{25}$ P. J. Reid and P. F. Barbara, J. Phys. Chem. 99, 3554 (1995).
${ }^{26}$ G. C. Walker, E. Akesson, A. E. Johnson, N. E. Levinger, and P. F. Barbara, J. Phys. Chem. 96, 3728 (1992).

${ }^{27}$ E. Akesson, A. E. Johnson, A. E. Levinger, G. C. Walker, T. P. Du Bruil, and P. F. Barbara, J. Chem. Phys. 96, 7859 (1992).

${ }^{28}$ M. Bixon and J. Jortner, Chem. Phys. 176, 467 (1993).

${ }^{29}$ M. Bixon, J. Jortner, and J. W. Verhoeven, J. Am. Chem. Soc. 116, 7349 (1994).

${ }^{30}$ M. Bixon and J. Jortner, Ber. Bunsenges. Phys. Chem. 99, 295 (1995).

${ }^{31}$ P. F. Barbara, G. C. Walker, and T. P. Smith, Science 256, 975 (1992).

${ }^{32}$ G. Feher, T. A. Arno, and M. Y. Okamura, in The Photosynthetic Bacterial Reaction Center Structure and Dynamics, edited by J. Berton and A. Vermeglio (Plenum, New York, 1988), p. 271.

${ }^{33}$ G. R. Fleming, J.-L. Martin, and J. Breton, Nature (London) 333, 190 (1988).

${ }^{34}$ J. Breton and A. Vermeglio, The Photosynthetic Bacterial Reaction Center (Plenum, New York, 1988).

${ }^{35}$ K. Wynne, C. Galli, and R. M. Hochstrasser, J. Chem. Phys. 100, 4797 (1993).

${ }^{36}$ F. Zhu, C. Galli, and R. M. Hochstrasser, J. Chem. Phys. 98, 1042 (1993).

${ }^{37}$ S. Iwai, T. Kamata, S. Murata, T. Fukaya, T. Kodaira, F. Mizukami, M. Tachiya, K. Yamamoto, and T. Ohta, J. Chem. Phys. 110, 8687 (1999).

${ }^{38}$ K. Yoshihara, Adv. Chem. Phys. 107, 371 (1999).

${ }^{39}$ M. Sparpaglione and S. Mukamel, J. Chem. Phys. 88, 3263 (1988).

${ }^{40}$ N. Makri and D. E. Makarov, J. Chem. Phys. 102, 4600 (1995).

${ }^{41}$ N. Makri and D. E. Makarov, J. Chem. Phys. 102, 4611 (1995).

${ }^{42}$ R. D. Coalson, D. G. Evans, and A. Nitzan, J. Chem. Phys. 101, 436 (1994).

${ }^{43}$ D. G. Evans and R. D. Coalson, J. Chem. Phys. 102, 5658 (1995).

${ }^{44}$ D. G. Evans and R. D. Coalson, J. Chem. Phys. 104, 3598 (1996).

${ }^{45}$ D. G. Evans, A. Nitzan, and M. A. Ratner, J. Chem. Phys. 108, 6387 (1998), and references therein.

${ }^{46}$ W. T. Pollard, A. K. Felts, and R. A. Friesner, Adv. Chem. Phys. 93, 77 (1996).

${ }^{47}$ J. Rasaiah and J. Zhu, J. Chem. Phys. 98, 1213 (1991).

${ }^{48}$ S. J. Rosenthal, X. Xie, M. Du, and G. R. Fleming, J. Chem. Phys. 95, 4715 (1991).

${ }^{49}$ W. Jarzeba, G. Walker, A. E. Johnson, and P. F. Barbara, Chem. Phys. 57, 152 (1991).

${ }^{50}$ R. Jimenez, G. R. Fleming, P. V. Kumar, and M. Maroncelli, Nature (London) 369, 471 (1994).

${ }^{51} \mathrm{~N}$. Mataga and T. Kubota, Molecular Interactions and Electronic Spectra (Marcel Dekker, New York, 1970).

${ }^{52}$ P. F. Barbara and W. Jarzeba, Adv. Photochem. 15, 1 (1990).

${ }^{53}$ S. Mukamel, Principles of Nonlinear Optical Spectroscopy (Oxford University Press, New York, 1995).

${ }^{54}$ S. Iwai, S. Murata, and M. Tachiya, J. Chem. Phys. 109, 5963 (1998).

${ }^{55}$ N. Gayathri and B. Bagchi, J. Phys. Chem. 100, 3056 (1996).

${ }^{56}$ N. Gayathri and B. Bagchi, J. Phys. Chem. A 103, 8496 (1999).

${ }^{57}$ D. J. Bicout and A. Szabo, J. Chem. Phys. 109, 2325 (1998).

${ }^{58}$ A. B. Myers and R.A. Mathies, in Biological Applications of Raman Spectroscopy, edited by T. G. Spiro (Wiley, New York, 1987), Vol. 2.

${ }^{59}$ B. Hudson and R.J. Sension, in Vibrational Spectra and Structure, edited by H. D. Bist, J. R. Durig, and J. F. Sullivan (Elsevier, Amsterdam, 1989), p. 363.

${ }^{60}$ K. Tominaga, D. A. V. Kliner, A. E. Johnson, N. E. Levinger, and P. F. Barbara, J. Chem. Phys. 98, 1228 (1993).

${ }^{61}$ K. Schulten, Z. Schulten, and A. Szabo, Physica A 100, 599 (1980).

${ }^{62}$ A. Szabo, G. Lamm, and G. H. Weiss, J. Stat. Phys. 34, 225 (1984).

${ }^{63}$ K. L. Sebastian, Phys. Rev. A 46, R1732 (1992).

${ }^{64}$ A. Samanta and S. K. Ghosh, Phys. Rev. E 47, 4568 (1993).

${ }^{65}$ H. A. Kramers, Physica (Amsterdam) 7, 284 (1940).

${ }^{66}$ R. F. Grote and J. T. Hynes, J. Chem. Phys. 77, 3736 (1982).

${ }^{67}$ I. Rips and J. Jortner, J. Chem. Phys. 87, 2090 (1987).

${ }^{68}$ I. Rips and J. Jortner, J. Chem. Phys. 87, 6513 (1987).

${ }^{69}$ I. Rips and J. Jortner, J. Chem. Phys. 88, 818 (1988). 